\title{
Protective effect and mechanism of mesenchymal stem cells on heat stroke induced intestinal injury
}

\author{
LU WANG ${ }^{1,2}$, ZIHUI DENG ${ }^{3}$, RUI YUAN $^{1,2}$, YAN ZHAO $^{2}$, MENGMENG YANG $^{2}$, JIE HU $^{2}$, \\ YU ZHANG ${ }^{2}$, YUN LI ${ }^{1,2}$, FEIHU ZHOU ${ }^{2}$ and HONGJUN KANG ${ }^{2}$ \\ ${ }^{1}$ Medical School of Chinese PLA; ${ }^{2}$ Department of Critical Care Medicine, The First Medical Center, \\ Chinese PLA General Hospital; ${ }^{3}$ Department of Biochemistry, Graduate School, \\ Chinese PLA General Hospital, Beijing 100853, P.R. China
}

Received December 6, 2019; Accepted May 29, 2020

DOI: $10.3892 /$ etm.2020.9051

\begin{abstract}
Heat stroke (HS) is considered to be a severe systemic inflammatory reaction disease that is caused by high fever. The mortality of HS is high worldwide due to the lack of effective treatments. Presently, mesenchymal stem cells (MSCs) have been demonstrated to serve roles in inflammation and immune regulation. Therefore, the current study aimed to investigate the protective effect and mechanism of MSCs against the HS-induced inflammatory response and organ dysfunction. A rat model of HS was induced by a high-temperature environment and treated with MSCs via tail veins. The levels of molecular markers of organ function, inflammatory factors and chemokines were examined at days 1, 7, 14 and 28. Histological staining was performed on the intestines of rats and control groups, and the Chiu's scores of the two groups were compared. The results revealed that MSCs injection significantly reduced the mortality and inhibited the circulatory inflammatory response. Additionally, main organ function, such as in the liver and kidney, were significantly improved following MSCs infusion in HS rats. Furthermore, MSCs treatment significantly improved edema, necrosis and villus exfoliation of intestinal mucosa, and reduced the inflammatory response of intestinal tissue. These results indicated that MSC infusion had therapeutic effects on $\mathrm{HS}$ of rats by regulating the circulatory and intestinal inflammatory response. Moreover, MSCs may be able to protect
\end{abstract}

Correspondence to: Professor Hongjun Kang, Department of Critical Care Medicine, The First Medical Center, Chinese PLA General Hospital, 28 Fu Xing Road, Beijing 100853, P.R. China E-mail: doctorkang301@126.com

Abbreviations: MSCs, mesenchymal stem cells; IL-1 $\beta$, interleukin-1 $\beta$; IL-6, interleukin-6; IL-10, interleukin-10; TNF- $\alpha$, tumor necrosis factor- $\alpha$; PGE2, prostaglandin E2; TLR, Toll-like receptor

Key words: mesenchymal stem cells, heat stroke, systemic inflammatory response syndrome, intestines, shock, sepsis organ function and promote tissue repair in HS. The results of the current study indicated that MSCs may be used as a potential method to treat HS and the resulting organ dysfunction.

\section{Introduction}

Heat stroke (HS) is a severe and life-threatening disease, characterized by excessive hyperthermia and nervous system symptoms such as confusion, seizures or loss of consciousness (1). HS can be divided into two types: Labor HS (caused by high-intensity physical work) and non-labor HS (caused by a high-temperature environment) (1). With increased temperature caused by global warming, the number of reported HS cases has increased within the last two decades (1). According to its pathophysiological characteristics, HS is a type of high fever-associated systemic inflammatory response, which can lead to multiple organ dysfunction syndrome (MODS) and the appearance of encephalopathy, especially central nervous system injury (2).

The small intestines can prevent bacterial invasion and participate in the process of water balance and solute transport within the body (3). In the initiation stage of HS inflammation, high fever is conducive to endotoxin leakage from the intestinal mucosa to the systemic circulation (4). Heat stimulation and high-intensity physical activity can damage intestinal mucosal cells, resulting in tissue hypoxia, ATP depletion, acidosis and oxidative stress and intestinal mucosal barrier dysfunction $(4,5)$. Previous animal and cell studies have demonstrated that heat stress can induce tissue hyperthermia and decrease intestinal blood flow, which may subsequently lead to tight junction disorders and pro-inflammatory cytokine release, or further induce the systemic inflammatory response $(6,7)$. Under these circumstances, the inflammatory reaction can cause vascular endothelial damage, which can aggravate the damage to the intestinal mucosal barrier (8).

Mesenchymal stem cells (MSCs) serve a number of roles, including enhancing angiogenesis and neurogenesis by secreting nutrient factors, regulating the immune system and exhibiting anti-inflammatory effects (5). MSCs may exhibit immunomodulatory effects, which are based on the mechanisms cell contact and paracrine signaling, and involve the release of soluble inflammatory factors (6). In particular, MSCs have been indicated to stimulate immune regulatory 
factors, including interleukin (IL)-6, IL-10, prostaglandins, transforming growth factor- $\beta$ (TGF- $\beta$ ) and nitric oxide (7). Furthermore, regulatory $\mathrm{T}$ cell production can be promoted and their inhibitory effect increased by MSCs, which can lead to the inhibition of intestinal inflammation by inhibiting macrophage production and regulating macrophage phenotype via prostaglandin E2 (PGE2) (8). Furthermore, aside from their potent anti-inflammatory effects, MSCs have been demonstrated to accelerate tissue repair, mainly through promoting epithelial formation, granulation tissue formation and angiogenesis (9). It has been hypothesized that the early onset of HS is due to intestinal ischemia, which results in intestinal mucosal barrier function damage and leads to the induction of the intestinal flora and the systemic inflammatory response (10). Furthermore, studies have reported that MSCs improved intestinal mucosal barrier function by repairing tight junctions and increasing the number of regenerating crypts and zonula occludens 1 protein (11-13).

Previous studies investigating the use of stem cells for the treatment of diabetes (14), stroke (15) and brain or spinal cord injury (16) have made rapid progress. Previous studies have focused on the beneficial effects of stem cells on HS brain injury $(17,18)$; however, a small number of studies have focused on intestinal injury during the initiation of inflammation (19). The current study aimed to investigate whether MSCs serve a protective role in intestinal injury and systemic inflammation that is caused by heat stroke. The present study aimed to identify effective methods and references that can be used in the treatment of HS and the improvement of patient prognosis. In the current study, a rat model of HS was established. The study group received MSCs injection intravenously following the successful formation of the model, while the control group was injected with $0.3 \mathrm{ml}$ normal saline. By observing the survival rate, biochemical indicators and cytokine expression levels of the two groups and examining the pathological condition of intestinal mucosa at different time points, the results demonstrated whether MSCs serve a protective role in intestinal injury and systemic inflammation caused by heat stroke.

\section{Materials and methods}

Animals. A total of 90 mature (age, 8 weeks; weight, 180-220 g) and 20 immature (age, 4 weeks; weight, 60-80 g) male Sprague-Dawley rats were provided by the Experimental Animal Center of Chinese PLA General Hospital. The current research complied with the statement of relevant ethical standards (the Animal Research: Reporting of in vivo Experiments reporting guidelines) (20) and was approved by the Ethics Committee of the Chinese PLA General Hospital (approval no. 2017-X13-10). All animals were conducted in accordance with the National Institutes of Health Guidelines for Animal Care and Use (21). The animals were kept in cages at room temperature of $22-25^{\circ} \mathrm{C}$, air humidity of $40 \%$, air pressure of $101.325 \mathrm{kPa}$ and $12 \mathrm{~h}$ light/dark cycles. All animals had free access to common rat feed and water. At the end of the experiment, rats were euthanized by neck dislocation following anesthesia.

Adipose-derived MSCs isolation, culture and identification. As described previously $(22,23)$, MSCs derived from fat were isolated and purified from immature rats. A total of 3 rats were euthanized by neck dislocation at different time points (at days 1, 7, 14 and 28). Adipose tissues were separated from the groin and then sliced and digested with $0.05 \%$ trypsin and $0.1 \%$ collagen I. The digestive solution was filtered and centrifuged in 2,000 x g. Cells were washed twice with PBS and cultured at $37^{\circ} \mathrm{C}$ in humidified air with $5 \% \mathrm{CO}_{2}$ in low-glucose DMEM (Gibco; Thermo Fisher Scientific, Inc.) supplemented with 10\% FBS (Gibco; Thermo Fisher Scientific, Inc.), 80 units $/ \mathrm{ml}$ penicillin and $0.2 \mathrm{mg} / \mathrm{ml}$ streptomycin. MSCs were identified as described previously $(22,23)$. Immunophenotypes of MSCs were stained at room temperature for $15 \mathrm{~min}$ with Allophycocyanin-conjugated cluster of differentiation (CD) 90 (1:20; cat. no. 561409; BD Biosciences), fluorescein isothiocyanate (FITC)-conjugated CD44 (1:20; cat. no. 550974; BD Biosciences), R-phycoerythrin-conjugated CD54 (1:20; cat. no. 554970; BD Biosciences), FITC-conjugated CD45 (1:20; cat. no. 554877; BD Biosciences), FITC-conjugated CD34 (1:20; cat. no. 11-0341-82; eBioscience; Thermo Fisher Scientific, Inc.) and FITC-conjugated CD11b (1:20; cat. no. 554982; BD Biosciences) and analyzed using a BD Accuri C6 flow cytometry software system (version 1.0.264.21; BD Biosciences). Additionally, adipocyte differentiation of MSCs was detected by Oil Red O staining (Sigma-Aldrich; Merck KGaA) at room temperature for $1 \mathrm{~h}$, and osteoblast differentiation was detected by Alizarin Red staining (Sigma-Aldrich; Merck KGaA) at room temperature for $30 \mathrm{~min}$. The cells were observed using an inverted light microscope (magnification, x40; Olympus BX53; Olympus Corporation). Freshly harvested early passage MSCs were used in all subsequent experiments.

HS injury rat model induction and treatment. The HS damage model was established as described previously $(24,25)$. A total of 5 mature rats at a time were kept at $40^{\circ} \mathrm{C}$ and $40 \%$ humidity. The rectal temperature of rats was continuously measured. When rectal temperatures reached $42^{\circ} \mathrm{C}$, the general condition including hind limb weakness and lethargy of the rats was observed. All rats were restored to room temperature and were given a peritoneal injection of $5 \mathrm{ml}$ saline. The HS injury model rats were divided into control (HS) and study (HS + MSCs) groups ( $n=40$ per group). After the model was established successfully, 2x10 $10^{6}$ MSCs suspended in $0.3 \mathrm{ml}$ of saline were injected into the HS+MSCs group and $0.3 \mathrm{ml}$ of saline were injected into the HS group via the tail vein. Rats in the HS and HS + MSCs groups were further divided into early (day 1), intermediate (days 7 and 14) and late (day 28) groups ( $n=10$ per group). Rats in the normal control group $(n=10)$ were not exposed to the high temperature and injected with $0.3 \mathrm{ml}$ of saline. All rats were observed for 28 days following HS injury with or without MSCs infusion to estimate survival rate.

Determination of the effects of infused MSCs on cytokines and biochemical markers in rats with HS injury. To detect the level of inflammatory factors [IL-1 $\beta$, IL-6, IL-10 and tumor necrosis factor- $\alpha$ (TNF- $\alpha)$ ] and chemokines [eotaxin and regulated upon activation normal $\mathrm{T}$ cell expressed and secreted (Rantes)], blood ( $n=5$ per group) and intestinal tissues ( $n=5$ per group) were collected on day 1 (early), days 7 and 14 (intermediate) and day 28 (late) following MSCs infusion and following the rats being euthanized. The blood samples were centrifuged at $2,000 \mathrm{xg}$ for $10 \mathrm{~min}$ at $4^{\circ} \mathrm{C}$ and the supernatant 

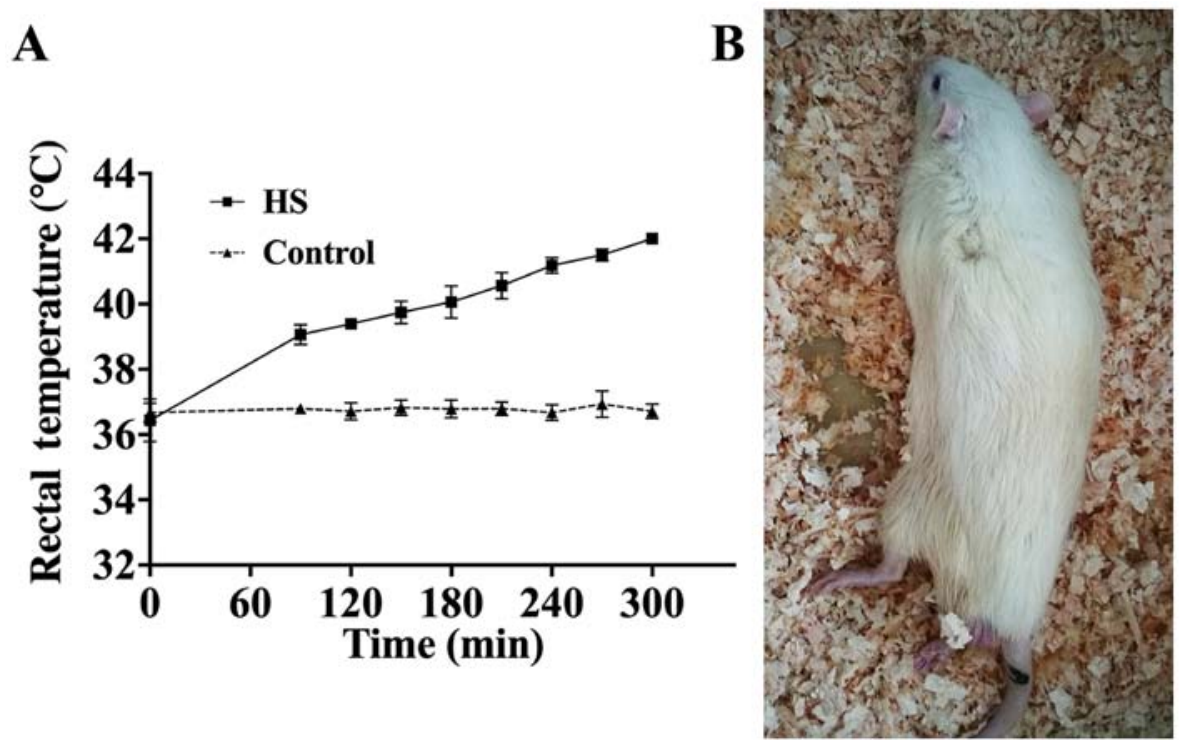

Figure 1. Modeling of HS in rats. (A) Temperature changes in the rats during the modeling process. (B) Following successful modeling, rats with HS exhibited hindlimb paralysis. HS, heat stroke.

was harvested. Brain samples were homogenized in a 10 -fold volume of cold PBS. The homogenate was centrifuged at $12,000 \mathrm{x}$ g for $15 \mathrm{~min}$ at $4^{\circ} \mathrm{C}$ and the supernatant was kept at $-80^{\circ} \mathrm{C}$ until subsequent measurement. Procarta Plex ${ }^{\mathrm{TM}}$ Analyst software (version 1.0; eBioscience; Thermo Fisher Scientific, Inc.) was used to determine the concentration of inflammatory factors and chemokines in blood and tissue lysates. For biochemical determination, blood samples were collected on day 1 (early), days 7 and 14 (intermediate), and day 28 (late) following MSCs or saline infusion for every group. Blood samples were centrifuged at 2,000 x g for $10 \mathrm{~min}$ at $4^{\circ} \mathrm{C}$ and the supernatant was collected for determination. The ALT (alanine aminotransferase), AST (aspartate aminotransferase), ALP (alkaline phosphatase), LDH (lactate dehydrogenase), CREA (creatinine) and UA (uric acid) were determined using an automated biochemical analyzer (7170-A; Hitachi, Ltd.).

Histological examinations. Intestinal tissue specimens were prepared by perfusion fixation with $4 \%$ paraformaldehyde (Wuhan Servicebio Technology Co., Ltd) at room temperature on days 1, 7, 14 and 28 following MSCs or saline infusion ( $n=3$ in each group), until the liver turned white, the samples were collected and eluted three times using PBS. Intestinal tissues were dissected and immersed in $4 \%$ paraformaldehyde (Wuhan Servicebio Technology Co., Ltd.) for $12 \mathrm{~h}$ initially and then immersed in $30 \%$ sucrose solution for $24 \mathrm{~h}$ at room temperature. The tissues were implanted in a cooled embedding medium (optimal cutting temperature solution; Sakura Finetek USA, Inc.). Following immediate freezing, tissues were cut using a frozen section machine (Leica Microsystems) into $7 \mu \mathrm{m}$ thick slices for dyeing. The sections were stained with hematoxylin and eosin, as in previous studies (26). Stained sections were visualized and scanned using a Pannoramic MIDI CaseViewer 2.0 System (3DHISTECH Ltd.). According to Chiu et al (27), the degree of injury to the intestinal tissues was evaluated, with each degree of injury ranging from 0 to 5 points.
Statistical analysis. SPSS software (version 23.0; IBM Corp.) was used to the analyze data. All experiments were conducted independently in triplicate. Data is expressed as mean \pm standard deviation. A Kolmogorov Smirnov test was used to assess whether the data was normally distributed, and a Levene test was used to analyze the homogeneity of variance. ANOVA and Tukey's test was used for continuous variables subject following data tests of normality and equivariance. Kaplan-Meier method was used for survival analysis of rats in each group. Otherwise, nonparametric statistical analysis (Mann-Whitney U test) was conducted for Chiu's score of intestinal tissue. $\mathrm{P}<0.05$ was considered to indicate a statistically significant difference.

\section{Results}

Establishment of the rat heat stroke model. As reported by previous studies $(22,23)$, modeling was considered to be complete once the rats exhibited symptoms of heat stress (including hind limb weakness and lethargy) and when rectal temperatures reached $42^{\circ} \mathrm{C}$. Real time rectal temperature changes in rats were monitored to identify a raised temperature of $42^{\circ} \mathrm{C}$ (Fig. 1A) and 80 rat models of heat stroke were obtained. The normal state of the rats (body temperature $42^{\circ} \mathrm{C}$ ) after modeling is presented in Fig. 1B.

Identification of MSCs. MSCs injected into the rats were identified by flow cytometry and differentiation ability. Following immunophenotyping, these MSCs were positive for CD90, CD44 and CD54, and negative for CD11b, CD45 and CD34 (Fig. 2A). Oil Red O and Alizarin Red staining demonstrated that the cells exhibited adipogenic and osteogenic differentiation (Fig. 2B).

Effects of MSCs on survival rate and organ function. Rats underwent necessary rescue measures simultaneously in the study (HS + MSCs) and control (HS) groups. The results 

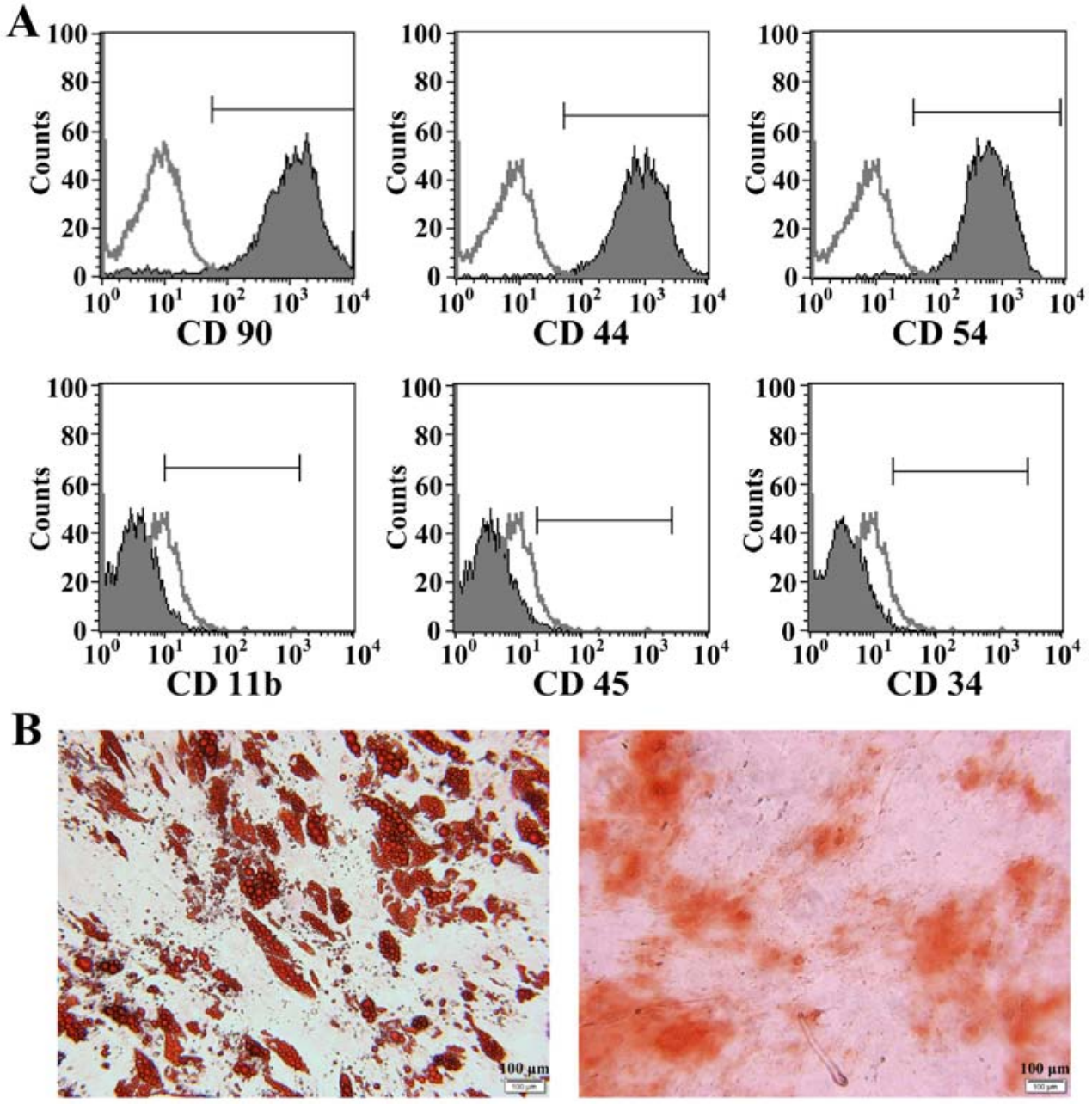

Figure 2. Characteristics of adipose-derived MSCs. MSCs were identified by their phenotypes and potential for differentiating into adipocytes and osteoblasts (A) Following immunophenotyping, these MSCs were positive for CD90, CD44 and CD54, and negative for CD11b, CD45 and CD34. (B) Differentiation of MSCs into adipocytes and osteoblasts was confirmed by oil red O staining (left image) and Alizarin Red staining (right image), respectively. Scale bar, $100 \mu \mathrm{m}$. MSCs, mesenchymal stem cells; $\mathrm{CD}$, cluster of differentiation.

demonstrated in the study group, the time required for rats to lower to normal body temperature was shorter (Fig. 3A) and mortality was significantly reduced (Fig. 3B) compared with the control group. Survival analysis indicated that the 28-day survival rate of the study group was significantly higher compared with the control group (Fig. 3C). At $24 \mathrm{~h}$ post-infusion of MSCs, the levels of biochemical indicators alanine aminotransferase (ALT), aspartate aminotransferase (AST), alkaline phosphatase (ALP), lactate dehydrogenase (LDH), creatinine (CREA) and uric acid (UA) in each group were detected. Compared with the control group, the level of all biochemical indicators in the study group were significantly improved, and the level of biochemical indicators in the normal group was lower compared with the other two groups (Fig. 3D-I).

Effects of MSCs on inflammatory factors and chemokines in blood. The levels on proinflammatory cytokines IL-1 $\beta$, IL-6 and TNF- $\alpha$ were significantly decreased compared with the control group (Fig. 4A, B and D) and the level of pro-inflammatory factors in the study group was obviously lower compared with the control group. TNF- $\alpha$ levels were significantly decreased in the study group compared with the control group (Fig. 4D). Furthermore, the level of IL-10 in the study group was significantly increased in a time-dependent manner compared with the control group, approaching normal levels at day 28 (Fig. 4C). The blood level of Rantes in the study group was lower at day 1 compared with the control group, then increased gradually. By day 7 the level of Rantes was significantly higher compared with the control group (Fig. 4E). Accordingly, the level of eotaxin in the study group peaked on day 1 , then gradually decreased on the day 14 , and increased gradually on day 28 (Fig. 4F). However, the overall level of eotaxin remained markedly higher compared with the control group at all subsequent time-points.

Intestinal histology. The pathological changes in the intestines of rats in each group are presented in Fig. 5. Following the successful establishment of the control group, numerous epithelial layers were separated from the lamina propria on both sides of the small intestinal villi and parts of the top of the villi were damaged. Additionally, there was an accumulation of inflammatory cells beneath the endothelium in the control group (Fig. 5A). According to Chiu's scores, intestinal injury gradually worsened from day 1 to day 7 (Fig. 5B). The highest score was $4.88 \pm 0.12$ at day 7 in the control group, after which pathological injury gradually decreased. At day 28 , 
A

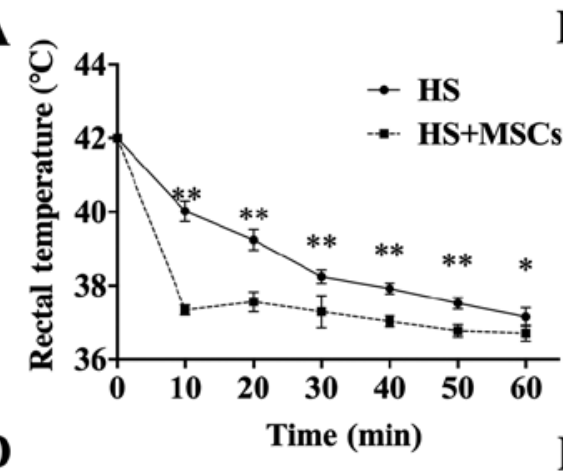

D
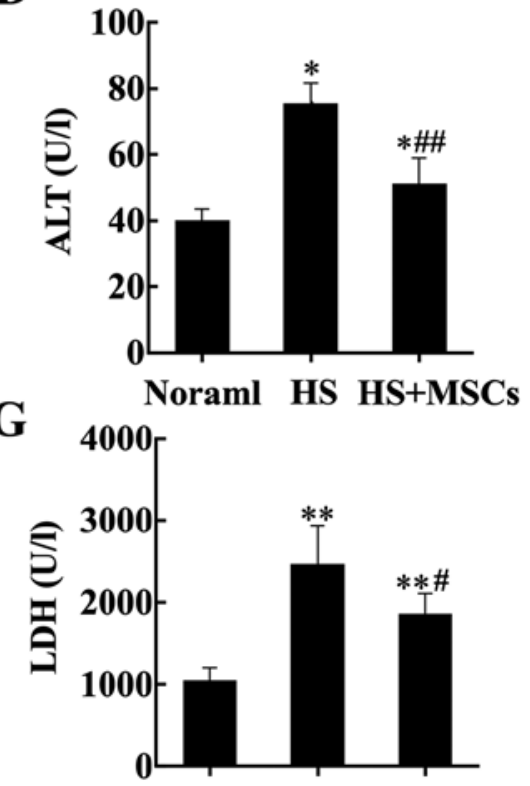

Noraml HS HS+MSCs
B

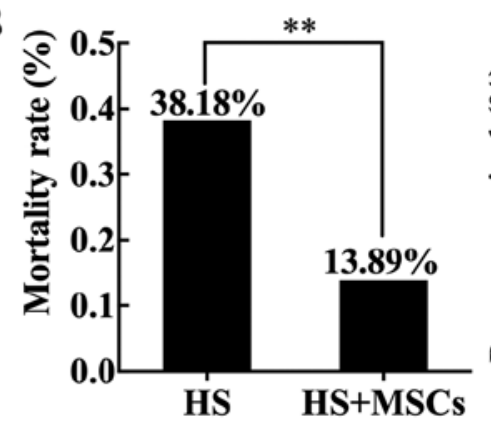

E

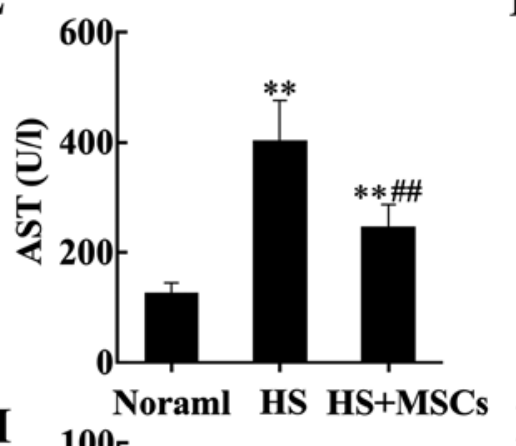

$\mathbf{F}$

C
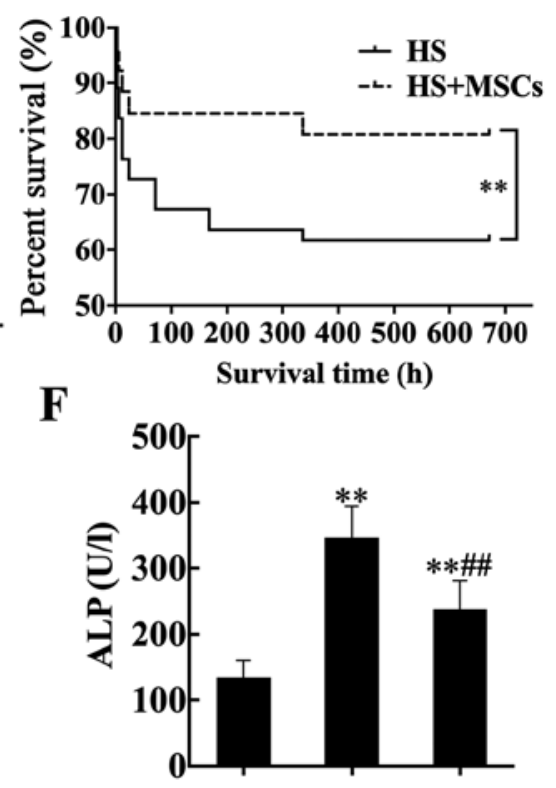

Noraml HS HS+MSCs

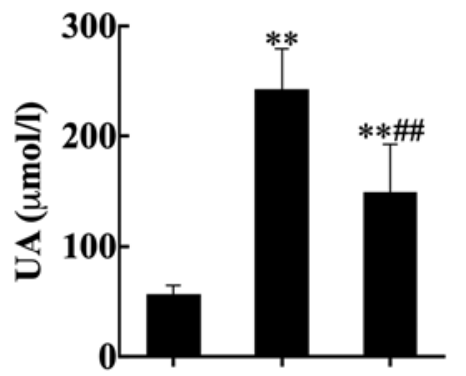

Noraml HS HS+MSCs

Figure 3. Cooling process, survival analysis and detection of organ markers. (A) Following the establishment of the model, rats were subjected to the temperature changes of rats were monitored and recorded. (B) Mortality rates were compared between the HS and HS + MSCs groups. (C) Survival analysis was performed for 28 days. Serum markers reflecting organ functions were measured, including (D) ALT, (E) AST, (F) ALP, (G) LDH, (H) CREA and (I) UA $(\mathrm{n}=5)$ and the results were analyzed. ${ }^{*} \mathrm{P}<0.05$ and ${ }^{* *} \mathrm{P}<0.01$ vs. normal group. ${ }^{\#} \mathrm{P}<0.05$ and ${ }^{\# \#} \mathrm{P}<0.01$ vs. control group. HS, heat stroke; MSCs, mesenchymal stem cells; ALT, alanine aminotransferase; AST, glutamic oxalate aminotransferase; ALP, alkaline phosphatase; LDH, lactate dehydrogenase; CREA, creatinine; UA, uric acid.

Chiu's score was $3.26 \pm 0.26$. The trend of variation of intestinal pathological scores in the study group were similar compared with the control group. However, pathological damage in the study group was significantly improved compared with the control group.

Effects of MSCs on inflammatory factors and chemokines in intestinal tissues. The level of IL-1 $\beta$ in the study group was significantly lower compared with the control group at the early stage (day 1 ), but the level of IL-1 $\beta$ in the control group began to decrease after day 1 , and was significantly lower than that in the study group on day 7, until day 28 (Fig. 6A). The levels of pro-inflammatory factors, including IL- 6 and TNF- $\alpha$, in the intestinal tissues of the study group were significantly lower compared with the control group (Fig. 6B and D). The trend of variation of IL-1 $\beta$ between groups was similar (Fig. 6A); however, the highest level of IL-1 $\beta$ in the study group was not similar to that in the control group and reverted to a normal level earlier than the control group. The difference in IL-6 and TNF- $\alpha$ levels between groups was evident at the early stage; however, the overall levels of both were lower in the study group compared with the control group (Fig. 6B and D). Furthermore, compared with the control group, the levels of anti-inflammatory factor IL-10 in the study group increased significantly at the early stage (day 1 and day 7; Fig. 6C). Chemokines, including Rantes and eotaxin, were detected in intestinal tissues (Fig. 6E and F). The level of Rantes in the early stage (day 1) in the study group was significantly higher compared with the control group, but on the day 7 and 14, the levels of Rantes in the study group were significantly lower compared with the control group. Furthermore, while the trend of variation of eotaxin was similar between groups, eotaxin levels in the study group was markedly lower compared with the control group from day 1 . The results therefore indicated that the levels of Rantes and eotaxin in the intestinal tissue were higher compared with the peripheral blood.

\section{Discussion}

At present, it is hypothesized that the intestine is the organ that initiates the systemic inflammatory response to heat stroke (10). High heat stimulation and intense exercise can lead 
A

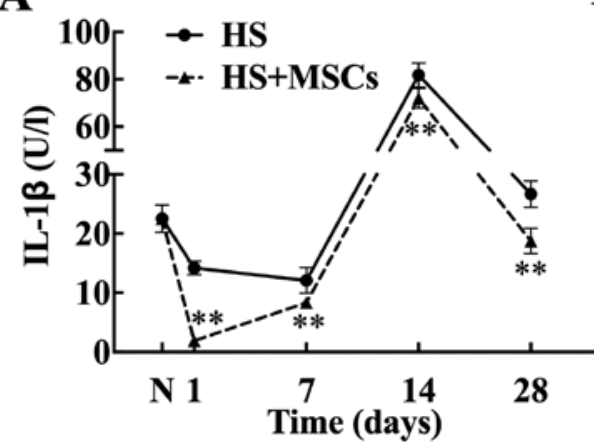

C

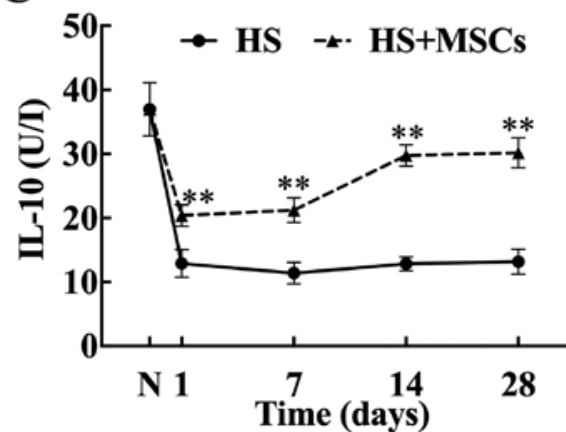

$\mathbf{E}$

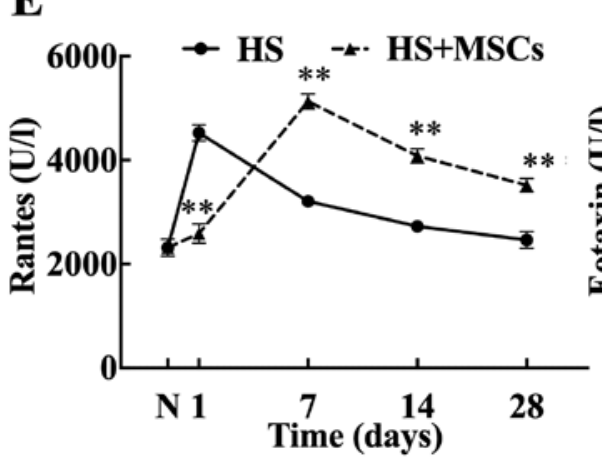

B

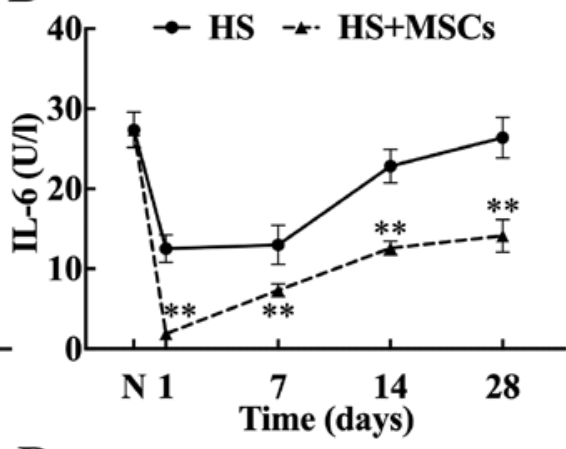

D

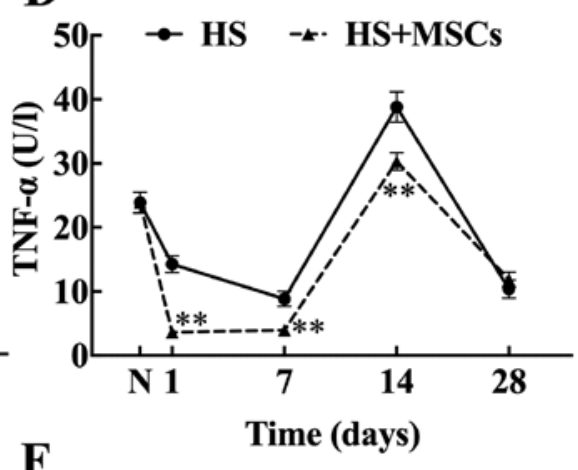

$\mathbf{F}$

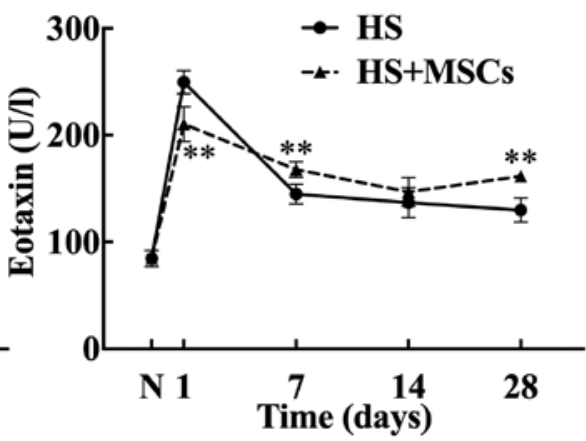

Figure 4. Inflammatory cytokines and chemokines in blood. The levels of inflammatory cytokines and chemokines in serum were measured on days 1, 7, 14 and 28 and plotted into a broken-line graph. The proinflammatory cytokines tested included (A) IL-1 $\beta$, (B) IL-6 and (D) TNF- $\alpha$ (n=5), and the anti-inflammatory cytokine tested was (C) IL-10 $(n=5)$. The chemokines detected were (E) Rantes $(n=5)$ and $(F)$ eotaxin $(n=5)$. These factors reflected the level of systemic inflammatory response. ${ }^{* * *} \mathrm{P}<0.01$ vs. control group. IL, interleukin; TNF- $\alpha$, tumor necrosis factor- $\alpha$; Rantes, regulated upon activation normal T cell expressed and secreted; HS, heat stroke; MSCs, mesenchymal stem cells; N, normal group.

to intestinal ischemia, intestinal mucosal cells damage and intestinal mucosal barrier dysfunction $(28,29)$. In addition to tissue hypoperfusion that is caused by hypotension, coagulation dysfunction also increases intestinal permeability, especially in tight junctions within the intestinal mucosal epithelium (28). Normal intestinal flora promotes digestion and absorption, protects liver and cardiovascular function (30), and improves iron metabolism (31). However, the destruction of the intestinal mucosal barrier causes intestinal flora and endotoxins to enter into the circulation (4), resulting in a systemic inflammatory response and MODS.

Hyperthermia can cause a decrease in intestinal blood flow, damage to intestinal mucosa and loss of tight junction integrity (10). Lambert et al (32) observed that the intestinal permeability of fluorescein isothiocyanate-glucose increased in anesthetized rats with core temperatures of $42.5^{\circ} \mathrm{C}$, and the pathological changes of the small intestine were clearer than those in the colon. A study also demonstrated that transmission electron microscopy revealed that intestinal epithelial cells were damaged, microvilli were lost, tight junctions were opened and mitochondria were swollen and vacuolated (32). Similarly, the current study demonstrated that following successful heat stroke modeling, numerous epithelial layers were separated from the lamina propria on both sides of the small intestinal villi and part of the tops of the villi were damaged, accompanied by accumulation of inflammatory cells beneath the endothelium. The results also revealed that the damage to intestinal mucosa was the most serious in the heat shock group at day 7 in the control group, with a Chiu's score of $4.88 \pm 0.12$. The disruption of intestinal mucosal barrier function leads to immense endotoxin entry into the circulation, which increases the production and release of pro-inflammatory factors, including IL-6, IL- $1 \beta$ and TNF- $\alpha$, activates endothelial cells, stimulates the release of endothelial activating factors and induces local or systemic inflammation (33). The results of the current study demonstrated that the levels of IL-1 $\beta$, IL-6 

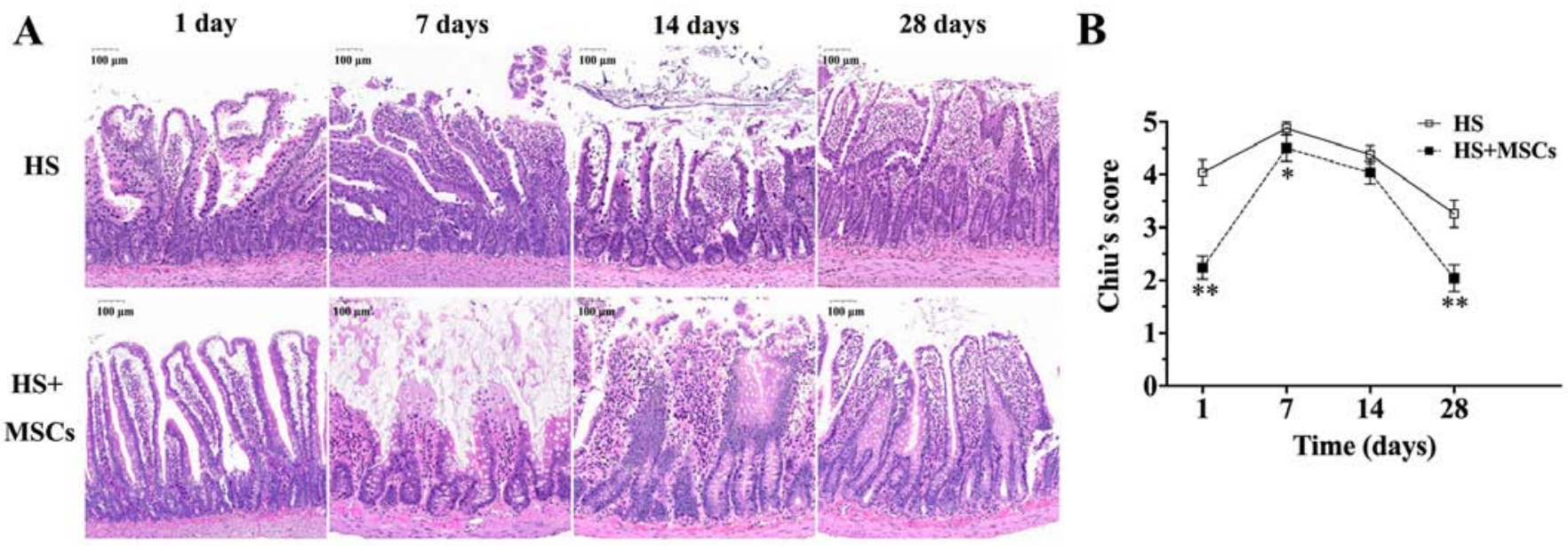

Figure 5. Histological examination and scoring of the intestine. (A) The small intestines of rats in the control and study groups were excised for histological examination on days 1,7,14 and 28. Intestinal tissue damage was evaluated at each time point according to Chiu's score and a (B) broken line chart (n=3) was drawn. Scale bar, $100 \mu \mathrm{m} .{ }^{*} \mathrm{P}<0.05$ and ${ }^{* *} \mathrm{P}<0.01$ vs. control group. HS, heat stroke; MSCs, mesenchymal stem cells.

A
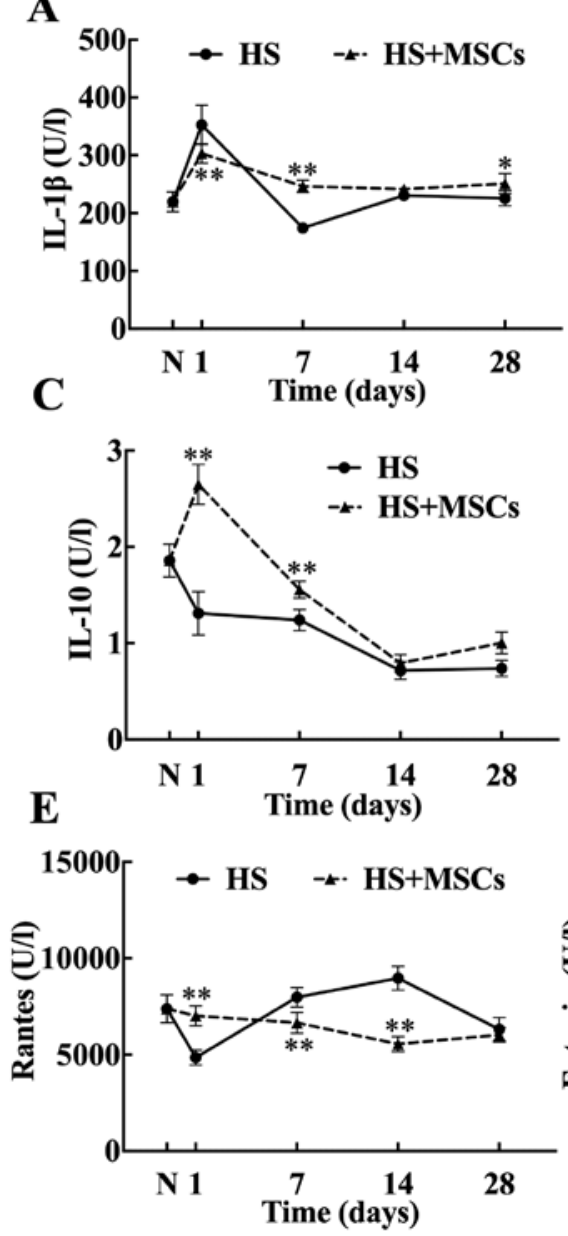

B
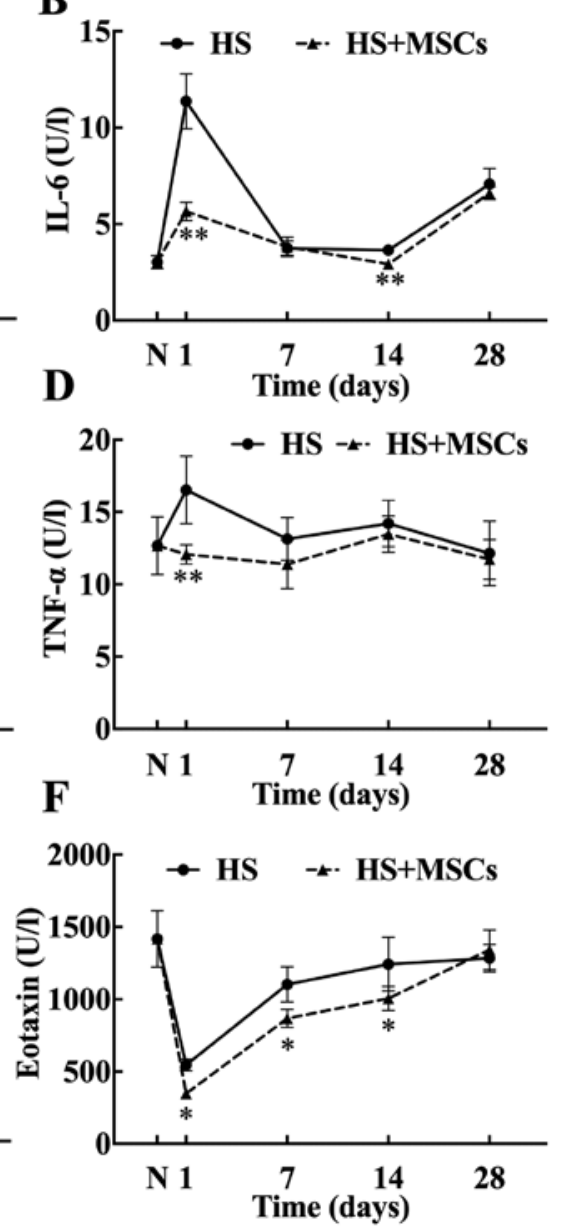

Figure 6. Inflammatory cytokines and chemokines in the intestinal tissues. The levels of inflammatory cytokines and chemokines in the intestinal tissues were measured on days 1, 7, 14 and 28 and plotted into a broken-line graph. The proinflammatory cytokines tested were (A) IL-1 $\beta$, (B) IL-6 and (D) TNF- $\alpha$ (n=5), and anti-inflammatory cytokine tested was (C) IL-10 (n=5). The chemokines detected were (E) Rantes $(n=5)$ and $(F)$ eotaxin $(n=5)$. These factors reflected the level of inflammatory response in intestinal tissues. ${ }^{*} \mathrm{P}<0.05$ and ${ }^{* *} \mathrm{P}<0.01$ vs. control group. IL, interleukin; TNF- $\alpha$, tumor necrosis factor- $\alpha$; Rantes, regulated upon activation normal T cell expressed and secreted; HS, heat stroke; MSCs, mesenchymal stem cells; N, normal group.

and TNF- $\alpha$ in intestinal tissues of the rats in the control group increased to varying degrees at day 1 following successful modeling.
MSCs have numerous functions, including regulating immune function and repairing tissue regeneration $(6,9)$. MSCs used in the current study were positive for CD90, 
CD44 and CD54, negative for CD11b, CD45 and CD34. Oil red $\mathrm{O}$ and alizarin red staining showed that the cells had adipogenic and osteogenic differentiation. A previous study has demonstrated that human umbilical cord blood stem cells improved the prognosis of heat stroke by reducing circulatory shock, improving brain injury and regulating inflammatory response (34). Similarly, a previous study has demonstrated that the application of human umbilical cord blood stem cells increased IL-10 levels and reduce TNF- $\alpha$ levels in the serum of mice with HS (18). Furthermore, a previous study has demonstrated that by using peripheral circulation measurement, MSCs improved the anti-inflammatory effect in sepsis to a certain degree, improve function of organs, including the lungs and kidneys, reduce the protein expression of inflammatory biomarkers and ultimately reduced the mortality of sepsis rats (35). Additionally, Weil et al (36) revealed that MSCs treatment prominently reduced the level of endotoxin-induced left ventricular myocardial inflammation biomarkers. In the current study, mortality statistics and survival analysis revealed that MSCs significantly improve the survival rate of HS rats. HS can cause multiple organ failure (2). The levels of certain organ markers were examined and the results reported that the levels of ALT, AST, ALP, LDH, CERA and UA in MSCs-treated rats decreased significantly, indicating that MSCs exhibited an effective protective effect on the liver and kidneys.

As the largest direct barrier between the environment and the host environment, gastrointestinal mucosa serves a key role in the regulation of immune system and the acquisition of tolerance against dietary antigens and the intestinal microbiota (37). Intestinal mucosal barriers allow nutrient uptake and immune surveillance, while limiting the transport of potentially harmful antigens and microorganisms (37). Intestinal integrity and immune homeostasis can be maintained by the dynamic regulation of intestinal mucosal structure and molecular interactions (37). The main pathophysiological mechanism of sepsis and HS is the recruitment of immune cells to produce an overwhelming immune response (2). Pathogen-associated molecular patterns, such as lipopolysaccharides, peptidoglycan and bacterial DNA, as well as damage-related molecular patterns (DAMPs), including mitochondrial DNA, high-mobility histone B1 and serum amyloid A, are upregulated by the recruitment of neutrophils and macrophages (38). As a first line of defense, cells migrate to intestinal tissue and produce proinflammatory cytokines, which are clinically typical of local and systemic inflammation $(39,40)$. Additionally, apoptosis and necrosis damage mucosal epithelium and lead to a cycle of DAMP release, resulting in increased inflammation and an imbalance of mucosal homeostasis (41). Therefore, normal intestinal function is crucial, and the destruction of the intestinal mucosal barrier causes bacterial translocation, leading to serious systemic inflammatory response (37). Histological staining was performed on the intestinal tissues of rats and evaluated using Chiu's score. The results revealed that the condition of intestinal mucosa improved in the early stage (day 1 following model establishment), which may be related to the self-repair function of intestinal mucosa; however, the rapid release of inflammatory factors aggravated mucosal damage. Furthermore, the intestinal scores of HS rats treated with MSCs on day 1, 7, 14 and 28 were significantly lower than that of the control group. Therefore, MSCs was indicated to significantly improve intestinal mucosal damage and protect intestinal barrier function.

Inflammatory factors and chemokines in intestinal tissue and peripheral blood were detected in the study and control groups. The results of intestinal tissue demonstrated that the levels of pro-inflammatory factors in the intestine were elevated due to local inflammation. However, in the early stages, the levels of pro-inflammatory factors in the study group were significantly lower compared with the control group, indicating that MSCs inhibited local inflammation. Levels of pro-inflammatory factors in peripheral blood were decreased, which may be associated with the inflammation of pro-inflammatory factors in tissues. However, levels of pro-inflammatory factors were decreased following MSCs treatment compared with the control group, indicating that MSCs also inhibited systemic inflammation. The level of anti-inflammatory factors IL-10 in the intestinal tissues differed from levels in peripheral blood. In intestinal tissues, IL-10 levels decreased in the control group, compared with the study group, indicating that MSCs promoted the local release of anti-inflammatory factors. Furthermore, IL-10 levels in the peripheral blood of both groups decreased; however, levels in the study group were significantly higher compared with the control group, indicating that MSCs promoted the systemic release of anti-inflammatory factors. This implied that MSCs may serve an anti-inflammatory role by increasing the level of anti-inflammatory factors. Additionally, the levels of two chemokines, Rantes and eotaxin, in intestinal tissue and peripheral blood were measured, were detected and the results indicated that their levels in intestinal tissues were significantly higher compared with levels in the peripheral blood, indicating that chemokines may serve a more significant role in local tissues. In intestinal tissues, the level of eotaxin in the study group was significantly lower compared with the control group on the days 1, 7 and 14. An increase in eotaxin levels in the later stage was associated with its main role in the acute phase of inflammation. The level of Rantes in the study group decreased, which may be associated with the role of Rantes in the middle and late stages of inflammation. Therefore, it may be concluded that MSC treatment significantly reduced chemokine levels, thereby inhibiting local tissue inflammation.

The current study demonstrated that MSCs have a positive protective effect on intestinal damage caused by HS. Combined with the results of previous studies, the present study hypothesized that MSCs may play a role in intestinal protection through the following aspects: As macrophages are key cells in a variety of intestinal inflammatory diseases, MSCs secrete macrophage-regulating molecules, including indoleamine 2,3-dioxygenase, TGF- $\beta$ and PGE2, which further stimulate macrophages to produce IL-10 by stimulating the production of PGE2 (42). Toll-like receptors (TLRs) are activated by intestinal-derived bacterial products and immune cells when the body is damaged (43). The immunomodulatory effects of MSCs activate TLRs and regulate the metallothionein 1-matrix metallopeptidase/janus kinase/signal transduced and activator of transcription 3 signaling pathway induced by TLR-2/6 targeting neovascularization (44). TLR4 activates the endotoxin-induced innate immune system and the NF- $\mathrm{kB}$ 
pathway, further stimulating the expression of pro-inflammatory factors (45). MSCs reduce the level of TLR4, leading to the reduction of pro-inflammatory factors and alleviating intestinal inflammatory response (46). A previous study has demonstrated that MSCs inhibited the proliferation and activation of cluster of differentiation (CD) $4^{+} \mathrm{T}$ cells and the differentiation of CD4+ T cells into Th1 and Th17 cells (47). These inhibitory effects are associated with the increased secretion of CD4+CD25 + forkhead box (Fox) p3 + regulatory T cells (Treg) and IL-10 (47). A previous study on acute kidney injury reported that MSCs therapy effectively reduced the expression of IL-17-related chemokines in serum and kidney tissues, as well as the infiltration of renal neutrophils (48). This is consistent with the results of the intestinal tissues assessed in the current study. Furthermore, the previous study demonstrated that MSCs restored the balance between Th17 cells and CD4 + CD25 + Foxp3 + Treg cells in intestinal tissues (48). Therefore, the current study hypothesized that MSCs affected the differentiation and expression of $\mathrm{T}$ cells, thereby regulating local and systemic immune function. Additionally, previous studies have revealed that MSCs may activate the Wingless-related integration site/ $\beta$-catenin signaling pathway, which is required for G-protein coupled receptor (LGR) $5^{+}$ cell proliferation through secretory factors (12), significantly stimulate intestinal epithelial cell proliferation, increase the number of LGR $5^{+}$intestinal stem cells and increase intestinal angiogenesis, thus serving a protective role in intestinal mucosa and promoting injury repair (49).

The current study proposed the use of MSCs for the treatment of HS and reported that MSCs effectively alleviated the inflammatory response of the whole body and local tissue, and protected the intestinal mucosal barrier. The present study demonstrated the anti-inflammatory and protective effects of MSCs on organs and provided new theories and methods for the treatment of HS, which are required to improve the prognosis of patients with HS. However, the signaling pathways involved in the regulation of the inflammatory response and the protection of organ function via MSCs in HS was not elucidated and subsequent experiments are required. Additionally, currently, there are also no uniform criteria for assessing the timing or dose of MSCs treatment and further studies will need to be conducted to determine these.

In conclusion, the current study demonstrated that MSCs reduced the levels of pro-inflammatory factors, regulated immune status and alleviated intestinal injury in rats with HS. Therefore, MSCs improved the systemic inflammatory response and tissue damage caused by HS and ultimately reduced the mortality of rats with HS.

\section{Acknowledgements}

Not applicable.

\section{Funding}

HK received a grant from the National Natural Science Foundation of China (grant no. 81671966), the Beijing Municipal Natural Science Foundation (grant no. 7182155) and the Application Research and Achievement Extension of Clinical Characteristics in Chinese Capital Foundation (grant no. Z171100001017160).

\section{Availability of data and materials}

The datasets used and/or analyzed during the current study are available from the corresponding author on reasonable request.

\section{Authors' contributions}

HK designed the current study, raised funds and revised the manuscript. LW conducted experiments, data collection and analysis and wrote the manuscript. ZD performed certain experiment and provided guidance for data analysis. RY, YanZ and MY performed certain data collection and analysis. YL, YuZ and $\mathrm{JH}$ conducted certain experiments. FZ designed the study and supervised the research. All authors read and approved the final manuscript.

\section{Ethics approval and consent to participate}

The current research complied with the statement of relevant ethical standards (the Animal Research: Reporting of in vivo Experiments reporting guidelines) (20) and was approved by the Ethics Committee of the Chinese PLA General Hospital (approval no. 2017-X13-10).

\section{Patient consent for publication}

Not applicable.

\section{Competing interests}

The authors declare that they have no competing interests.

\section{References}

1. Alan N Peiris SJ and Rabiya N: Heat Stroke. JAMA 318: 2503 , 2017.

2. Epstein Y and Yanovich R: Heatstroke. N Engl J Med 380: 2449-2459, 2019.

3. Haussner F, Chakraborty S, Halbgebauer R and Huber-Lang M: Challenge to the intestinal mucosa during sepsis. Front Immunol 10: 891, 2019.

4. Lim CL and Mackinnon LT: The roles of exercise-induced immune system disturbances in the pathology of heat stroke the dual pathway model of heat stroke. Sports Med 36: 39-64, 2006.

5. Kalladka D and Muir KW: Brain repair: Cell therapy in stroke. Stem Cells Cloning 7: 31-44, 2014.

6. Mishra VK, Shih HH, Parveen F, Lenzen D, Ito E, Chan TF and Ke LY: Identifying the therapeutic significance of mesenchymal stem cells. Cells 9: 1145, 2020.

7. Wang Y, Chen X, Cao W and Shi Y: Plasticity of mesenchymal stem cells in immunomodulation: Pathological and therapeutic implications. Nat Immunol 15: 1009-1016, 2014.

8. Park HJ, Kim J, Saima FT, Rhee KJ, Hwang S, Kim MY, Baik SK, Eom YW and Kim HS: Adipose-derived stem cells ameliorate colitis by suppression of inflammasome formation and regulation of M1-macrophage population through prostaglandin E2. Biochem Biophys Res Commun 498: 988-995, 2018.

9. Fu X, Liu G, Halim A, Ju Y, Luo Q and Song AG: Mesenchymal stem cell migration and tissue repair. Cells 8: 784, 2019.

10. Pires W, Veneroso CE, Wanner SP, Pacheco DAS, Vaz GC, Amorim FT, Tonoli C, Soares DD and Coimbra CC: Association between exercise-induced hyperthermia and intestinal permeability: A systematic review. Sports Med 47: 1389-1403, 2017.

11. Zhang W, Shen ZY, Song HL, Yang Y, Wu BJ, Fu NN and Liu T: Protective effect of bone marrow mesenchymal stem cells in intestinal barrier permeability after heterotopic intestinal transplantation. World J Gastroenterol 20: 7442-7451, 2014. 
12. Gong W, Guo M, Han Z, Wang Y, Yang P, Xu C, Wang Q, Du L, Li Q, Zhao H, et al: Mesenchymal stem cells stimulate intestinal stem cells to repair radiation-induced intestinal injury. Cell Death Dis 7: e2387, 2016.

13. Te Winkel J, John QE, Hosfield BD, Drucker NA, Das A, Olson KR and Markel TA: Mesenchymal stem cells promote mesenteric vasodilation through hydrogen sulfide and endothelial nitric oxide. Am J Physiol Gastrointest Liver Physiol 317: G441-G446, 2019.

14. Davey GC, Patil SB, O'Loughlin A and O'Brien T: Mesenchymal stem cell-based treatment for microvascular and secondary complications of diabetes mellitus. Front Endocrinol (Lausanne) 5: 86, 2014.

15. Tobin MK, Bonds JA, Minshall RD, Pelligrino DA, Testai FD and Lazarov O: Neurogenesis and inflammation after ischemic stroke: What is known and where we go from here. J Cereb Blood Flow Metab 34: 1573-1584, 2014.

16. Penha EM, Meira CS, Guimaraes ET, Mendonça MV, Gravely FA, Pinheiro CM, Pinheiro TM, Barrouin-Melo SM, Ribeiro-Dos-Santos R and Soares MB: Use of autologous mesenchymal stem cells derived from bone marrow for the treatment of naturally injured spinal cord in dogs. Stem Cells Int 2014: 437521, 2014.

17. Hsuan YC, Lin CH, Chang CP and Lin MT: Mesenchymal stem cell-based treatments for stroke, neural trauma, and heat stroke. Brain Behav 6: e00526, 2016.

18. Tseng L, Chen S, Lin M and Lin Y: Umbilical cord blood-derived stem cells improve heat tolerance and hypothalamic damage in heat stressed mice. Biomed Res Int 2014: 685683, 2014.

19. Zhang XM, Zhang YJ, Wang W, Wei YQ and Deng HX: Mesenchymal stem cells to treat Crohn's disease with fistula. Hum Gene Ther 28: 534-540, 2017.

20. Kilkenny C, Browne WJ, Cuthill IC, Emerson M and Altman DG: Improving bioscience research reporting: The ARRIVE guidelines for reporting animal research. PLoS Biol 8: e1000412, 2010

21. NIH (National Institutes of Health U.S.A). Guide for the Care and Use of Laboratory Animals. The National Academies Press, Washington, D.C, pp246, 2011

22. Si Y, Zhao Y, Hao H, Liu J, Guo Y, Mu Y, Shen J, Cheng Y, Fu X and Han W: Infusion of mesenchymal stem cells ameliorates hyperglycemia in type 2 diabetic rats identification of a novel role in improving insulin sensitivity. Dibaetes 61: 1616-1625, 2012.

23. Deng Z, Xu H, Zhang J, Yang C, Jin L, Liu J, Song H, Chen G, $\mathrm{Han} \mathrm{W}$ and Si Y: Infusion of adiposederived mesenchymal stem cells inhibits skeletal muscle mitsugumin 53 elevation and thereby alleviates insulin resistance in type 2 diabetic rats. Mol Med Rep 17: 8466-8474, 2018.

24. Ji J, Gu Z, Li H, Su L and Liu Z: Cryptdin-2 predicts intestinal injury during heatstroke in mice. Int J Mol Med 41: 137-146, 2018.

25. Phillips NA, Welc SS, Wallet SM, King MA and Clanton TL: Protection of intestinal injury during heat stroke in mice by interleukin-6 pretreatment. J Physiol 593: 739-753, 2015.

26. Deng ZH Jr, Yan GT, Wang LH, Zhang JY, Xue H and Zhang K: Leptin relieves intestinal ischemia/reperfusion injury by promoting ERK1/2 phosphorylation and the NO signaling pathway. J Trauma Acute Care Surg 72: 143-149, 2012.

27. Chiu CJ, McArdle AH, Brown R, Scott HJ and Gurd FN: Intestinal mucosal lesion in low-flow States. I. A morphological, hemodynamic, and metabolic reappraisal. Arch Surg 101: 478-483, 1970.

28. Dokladny K, Moseley PL and Ma TY: Physiologically relevant increase in temperature causes an increase in intestinal epithelial tight junction permeability. Am J Physiol Gastrointest Liver Physiol 290: G204-G212, 2006.

29. Pals KL, Chang RT, Ryan AJ and Gisolfi CV: Effect of running intensity on intestinal permeability. J Appl Physiol 82: 571-576, 1997.

30. Skrypnik K, Bogdański P, Łoniewski I, Reguła J and Suliburska J: Effect of probiotic supplementation on liver function and lipid status in rats. Acta Sci Pol Technol Aliment 17: 185-192, 2018.

31. Skrypnik K, Bogdanski P, Schmidt M and Suliburska J: The effect of multispecies probiotic supplementation on iron status in rats. Biol Trace Elem Res 192: 234-243, 2019.
32. Lambert GP, Gisolfi CV, Berg DJ, Moseley PL, Oberley LW and Kregel KC: Selected Contribution: Hyperthermia-induced intestinal permeability and the role of oxidative and nitrosative stress J Appl Physiol 92: 1750-1761, 2002.

33. Yan YE,Zhao YQ, Wang H and Fan M: Pathophysiological factors underlying heatstroke. Med Hypotheses 67: 609-617, 2006.

34. Liu WS, Chen CT, Foo NH, Huang HR, Wang JJ, Chen SH and Chen TJ: Human umbilical cord blood cells protect against hypothalamic apoptosis and systemic inflammation response during heatstroke in rats. Pediatr Neonatol 50: 208-216, 2009.

35. Chang C, Leu S, Sung HC, Zhen YY, Cho CL, Chen A, Tsai TH, Chung SY, Chai HT, Sun CK, et al: Impact of apoptotic adipose-derived mesenchymal stem cells on attenuating organ damage and reducing mortality in Rat sepsis syndrome induced by cecal puncture and ligation. J Transl Med 10: 244, 2012.

36. Weil BR, Manukyan MC, Herrmann JL, Wang Y, Abarbanell AM, Poynter JA and Meldrum DR: Mesenchymal stem cells attenuate myocardial functional depression and reduce systemic and myocardial inflammation during endotoxemia. Surgery 148: 444-452, 2010

37. Romero ES, Cotoner CA, Camacho CP, Bedmar MC and Vicario $\mathrm{M}$ : The intestinal barrier function and its involvement in digestive disease. Rev Esp Enferm Dig 107: 686-696, 2015.

38. Bianchi ME: DAMPs, PAMPs and alarmins all we need to know about danger. J Leukoc Biol 81: 1-5, 2007.

39. Nascimento LO, Massari P and Wetzler LM: The role of TLR2 in infection and immunity. Front Immunol 3: 79, 2012.

40. Schnoor M, Garcia Ponce A, Vadillo E, Pelayo R, Rossaint J and Zarbock A: Actin dynamics in the regulation of endothelial barrier functions and neutrophil recruitment during endotoxemia and sepsis. Cell Mol Life Sci 74: 1985-1997, 2017.

41. Jiang LY, Zhang M, Zhou TE, Yang ZF, Wen LQ and Chang JX: Changes of the immunological barrier of intestinalmucosa in rats with sepsis. World J Emerg Med 1: 138-143, 2010.

42. Németh K, Leelahavanichkul A, Yuen PS, Mayer B, Parmelee A, Doi K, Robey PG, Leelahavanichkul K, Koller BH, Brown JM, et al: Bone marrow stromal cells attenuate sepsis via prostaglandin $\mathrm{E}(2)$-dependent reprogramming of host macrophages to increase their interleukin-10 production. Nat Med 15: 42-49, 2009.

43. Yiu JH, Dorweiler B and Woo CW: Interaction between gut microbiota and toll-like receptor: From immunity to metabolism. J Mol Med (Berl) 95: 13-20, 2017.

44. Zgheib A, Pelletier-Bonnier E, Levros LCJ and Annabi B: Selective JAK/STAT3 signalling regulates transcription of colony stimulating factor- 2 and -3 in Concanavalin-A-activated mesenchymal stromal cells. Cytokine 63: 187-193, 2013.

45. Poltorak A, He X, Smirnova I, Liu MY, Van Huffel C, Du X, Birdwell D, Alejos E, Silva M, Galanos C, et al: Defective LPS signaling in $\mathrm{C} 3 \mathrm{H} / \mathrm{HeJ}$ and $\mathrm{C} 57 \mathrm{BL} / 10 \mathrm{ScCr}$ mice: Mutations in Tlr4 gene. Science 282: 2085-2088, 1998.

46. Niu GC, Liu L, Zheng L, Zhang H, Shih DQ and Zhang X: Mesenchymal stem cell transplantation improves chronic colitis-associated complications through inhibiting the activity of toll-like receptor-4 in mice. BMC Gastroenterol 18: 127, 2018.

47. Crawford PL, Kurte M, Bravo-Alegría J, Contreras R, Nova-Lamperti E, Tejedor G, Noël D, Jorgensen C, Figueroa F, Djouad F and Carrión F: Mesenchymal stem cells generate a CD4+CD25+Foxp3+ regulatory $\mathrm{T}$ cell population during the differentiation process of Th1 and Th17 cells. Stem Cell Res Ther 4: 65, 2013.

48. Luo CJ, Zhang FJ, Zhang L, Geng YQ, Li QG, Hong Q, Fu B, Zhu F, Cui SY, Feng Z, et al: Mesenchymal stem cells ameliorate sepsis-associated acute kidney injury in mice. Shock 41: 123-129, 2014.

49. Soontararak S, Chow L, Johnson V, Coy J, Wheat W, Regan D and Dow S: Mesenchymal stem cells (MSC) derived from induced pluripotent stem cells (iPSC) equivalent to adipose-derived MSC in promoting intestinal healing and microbiome normalization in mouse inflammatory bowel disease model. Stem Cells Transl Med 7: 456-467, 2018.

This work is licensed under a Creative Commons Attribution-NonCommercial-NoDerivatives 4.0 International (CC BY-NC-ND 4.0) License. 